\title{
The Possibilities of Determining the Effective Thermal Conductivity of Steel Rectangular Section Bundles Based on the Selected Literature Models
}

\author{
Rafal Wyczolkowski and Agnieszka Benduch \\ The Faculty of Production Engineering and Materials Technology, Czestochowa University of Technology, Department of Industrial \\ Furnaces and Environmental Protection, 42-200 Czestochowa, Poland
}

\begin{abstract}
The article presents the results of calculations of the effective thermal conductivity $k_{\mathrm{ef}}$ for bundles of steel rectangular sections obtained for a few analytical models. This coefficient expresses the ability of the bundles to heat transfer. The knowledge about the values of the $k_{\text {ef }}$ coefficient of the section bundles is essential to correctly identify the parameters of their heat treatment process. The quality of the calculation results were verified by the experimental measurement data. These measurements were performed in the guarded hot plate apparatus. It should be noted, that none of the eleven analyzed models of effective thermal conductivity is suitable for evaluation of thermal properties of the section bundles.
\end{abstract}

Key words: Effective thermal conductivity, steel rectangular sections, heat treatment, porous charge.

\section{Nomenclature}

$\mathrm{k}_{\mathrm{ef}}$ effective thermal conductivity $(\mathrm{W} / \mathrm{m} \cdot \mathrm{K})$

$\mathrm{k}_{\mathrm{s}} \quad$ thermal conductivity for solid phase $(\mathrm{W} / \mathrm{m} \cdot \mathrm{K})$

$\mathrm{k}_{\mathrm{g}} \quad$ thermal conductivity for gas phase $(\mathrm{W} / \mathrm{m} \cdot \mathrm{K})$

q heat flux $\left(\mathrm{W} / \mathrm{m}^{2}\right)$

$t_{B} \quad$ temperature of the bottom surface $\left({ }^{\circ} \mathrm{C}\right)$

$\mathrm{t}_{\mathrm{U}} \quad$ temperature of the upper surface $\left({ }^{\circ} \mathrm{C}\right)$

$\mathrm{h}$ sample dimension in the direction of heat flow $(\mathrm{m})$

\section{Greek Symbols}

$\varphi \quad$ porosity of the medium

$v_{\mathrm{s}} \quad$ volumetric content of solid phase

\section{Introduction}

Rectangular sections belong to the basic long products supplied by steel industry. They are widely used in various areas of the economy where they are most often used as a feedstock for further processing. In many cases the heat treatment is one of the manufacturing stages of steel sections. These elements are most commonly treated in the form of bundles

Corresponding author: Rafal Wyczolkowski, Ph.D., research fields: heat transfer in porous media and heat transfer in metallurgical processes. which are one of examples of porous charge.

The industrial heat treatment operations of steel products have significant bearing on all the key performance metrics of the plant. These metrics primarily include: productivity, energy consumption, product quality and emission of pollutants $[1,2]$. Mentioned factors make that the operation of heat treatment should be optimized. What is important, this optimization should be done both at the designed stage of the process as well as during its realization at the technological line. Nowadays for the efficient process design and its control the special numerical models are used which have the capability of predicting the spatial and temporal changes of the temperature of treated charge. Such models were successfully used in the metallurgical industry for over last three decades [3, 4].

One of the parameters of such models, which is necessary for its proper operation are the thermal properties of the heated charge. When heating process of monolithic steel elements are analysed, the basic thermal property is the thermal conductivity of steel $k_{\mathrm{s}}$. 
This parameter depends on the composition, crystalline structure and temperature of steel. The data about $k_{\mathrm{s}}$ coefficient of the most popular carbon and alloy steels are commonly available in the thematic literature $[5,6]$.

However, section bundles considered in this article do not have a monolithic structure. From this reason its thermal property necessary to the optimization of the heat treatment is not a thermal conductivity of steel. The effective thermal conductivity $k_{\mathrm{ef}}$, as the main thermal property of section bundles, should be considered. The knowledge about the $k_{\mathrm{ef}}$ coefficient is essential for the selection of the optimal parameters for heating of section bundles. Incorrect selection of $k_{\text {ef }}$ coefficient of section bundle can lead to improper course of heating process, disturb furnace operation and unnecessarily increase the heating time.

This article is devoted to the issue of the effective thermal conductivity modelling of steel rectangular section bundles.

\section{Structure of Steel Section Bundles and Their Thermal Properties}

Due to their geometrical structure, steel section bundles represent a very specific type of charge. An example of a model of a section bundle is presented in
Fig. 1. Due to presence of free spaces filled with gas, this bundle represents a two-phase (solid-gas) porous medium. Gas phase fills internal spaces in individual sections and the narrow gaps between each other. Presence of these gaps results from rounding of section edges and geometry errors, such as errors of rectilinearity and surface flatness. With this structure, analyzed bundle should be considered as a medium with mixed porosity: internal and external. Internal porosity is constant and depends on geometry of sections (external dimensions and wall thickness), whereas external porosity depends on the packing of a bundle (gap width). Internal porosity can exceed even the worth of 0.85 , whereas external porosity in packed bundles does not exceed 0.03 .

Length of real section bundles heated during industrial heat treatment is determined by the length of sections supplied by manufacturers. This length ranges most frequently from 3 to $6 \mathrm{~m}$, while transverse dimensions of bundles usually do not exceed $1 \mathrm{~m}$. Due to the disproportion between transverse dimensions and length, the intensity of the bundle heating is determined by the processes of heat exchange which occurs in transverse direction. The bundle in this plane is characterized by the lack of continuity of the solid phase, which allows for considering

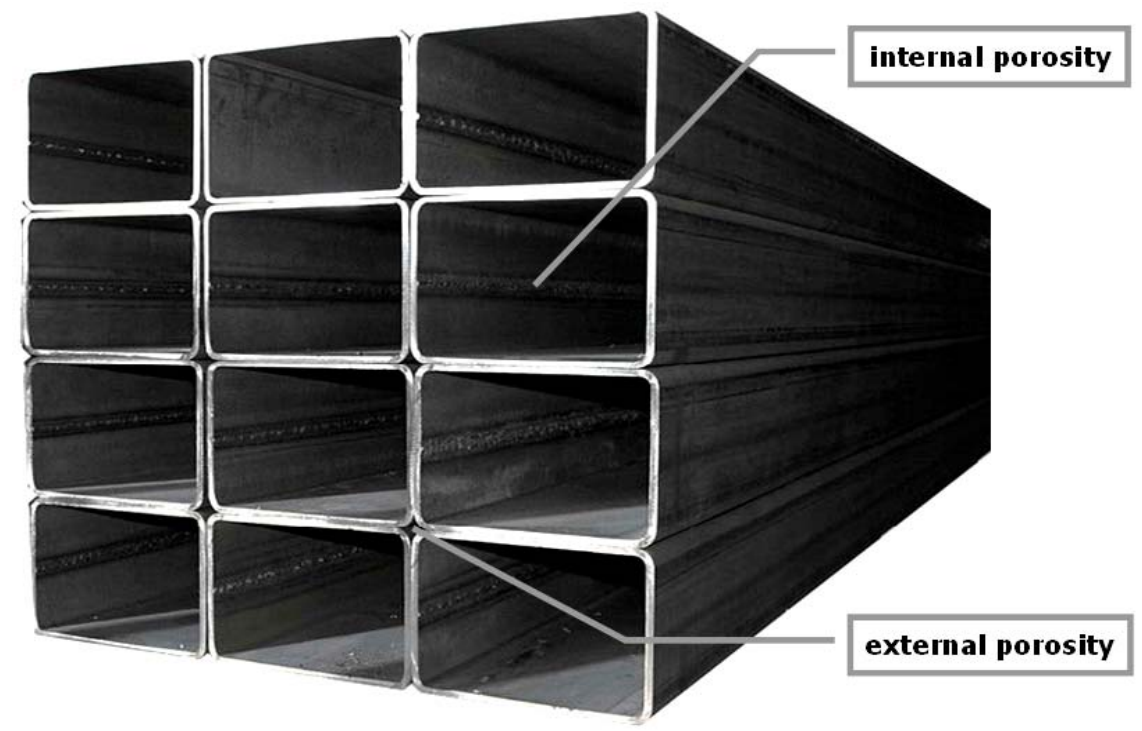

Fig. 1 Model of packed bundle of rectangular sections. 
it as a granular medium. During the heating process, thermal energy in this system is transferred through the following mechanisms:

- conduction along walls of individual sections, which corresponds to conduction in the solid phase;

- conduction in the gas which fills the interior of sections;

- conduction in the gas which fills the gaps between individual sections;

- thermal radiation between internal surfaces of sections;

- thermal radiation between external surfaces of adjacent sections;

- free convection of gas inside profiles;

- contact conduction at the locations of contact of adjacent sections.

Due to this complexity, the ability of the bundles to heat transfer in the transverse direction is expressed by means of the effective thermal conductivity $k_{\mathrm{ef}}$. This parameter is commonly used in the theory of porous media $[7,8]$. Value of the coefficient $k_{\text {ef }}$ for a specific section bundle depends on many factors, which include: thermal conductivity of steel $k_{\mathrm{s}}$, thermal conductivity of gas $k_{\mathrm{g}}$, dimensions of section, packing of the bundle, pressing force and state of section surface.

\section{Models of Effective Thermal Conductivity}

Several various theoretical models have been used in order to calculate the effective thermal conductivity of porous materials $[9,10]$. Below are presented several most frequently used and discussed models, which, in order to calculate the coefficient $k_{\text {ef }}$, take into consideration the porosity of the medium $\varphi$, volumetric content of solid state $v_{\mathrm{s}}$ and thermal conductivity of the solid phase $k_{\mathrm{s}}$ and gas phase $k_{\mathrm{g}}$.

The least complex models are layer models, where porous medium is considered as a system of alternate flat layers of solid and gas phases. Depending on the direction of heat flow with respect to these phases, are obtained two models: parallel (P) and series (S) [11]:

$$
\begin{gathered}
k_{e f}^{P}=(1-\varphi) \cdot k_{s}+\varphi \cdot k_{g} \\
k_{e f}^{S}=\left(\frac{1-\varphi}{k_{s}}+\frac{\varphi}{k_{g}}\right)
\end{gathered}
$$

These models refer to extreme cases of heat flow in two-phase porous medium. For this reason, coefficients $k_{\text {ef }}$ calculated according to the series and parallel model determine lower and upper limit of effective thermal conductivity of the material with porosity $\varphi$, and thermal conductivity for solid phase $k_{\mathrm{s}}$ and gas phase $k_{\mathrm{g}}$. This is given by the following inequality:

$$
k_{e f}^{S} \leq k_{e f} \leq k_{e f}^{P}
$$

Effective conductivity obtained for the parallel and series models has been used in other analytical models proposed by Horai (H) [12], Beck (B) [13] and Krischer (KR) [14] which are described with the following equations:

$$
\begin{gathered}
k_{e f}^{H}=\frac{k_{e f}^{P}+k_{e f}^{S}}{2} \\
k_{e f}^{B}=\left(k_{e f}^{P} \cdot k_{e f}^{S}\right)^{0.5} \\
k_{e f}^{K R}=\left(\frac{1-f}{k_{e f}^{P}}+\frac{f}{k_{e f}^{S}}\right)^{-1}
\end{gathered}
$$

Coefficient $k_{\mathrm{ef}}$ calculated according to the Horai model represents an arithmetic mean and, according to the Beck model, a geometric mean of the values obtained based on the models $\mathrm{P}$ and $\mathrm{S}$. The problem looks different in the case of the Krischer model. The parameter $f$ used in this model adopts the values ranging from $0 \div 1$. A parallel model is obtained for $f=$ 0 , whereas a series model is obtained for $f=1$. Depending on the choice of parameter $f$ for constant values $k_{\mathrm{s}}, k_{\mathrm{g}}$ and $\varphi$, very different values of effective conductivity are obtained. Therefore, this model seems to be very elastic and convenient in practical use. However, for the specified porous medium, the proper value of $f$ parameter can be obtained based 
only on the experimental measurements. This fact causes that this model has only theoretical character.

Other models of effective thermal conductivity are ME (Maxwell-Euken) models [9, 11]. They are introduced with the assumption that, in the structure of porous medium, one of the phases is dispersed, creating discrete particles in the area of the second phase, which has a continuous character. There are two cases, and, consequently, two ME models. The first Maxwell-Euken model (ME1) concerns cellular material where gas phase is dispersed in the solid phase:

$$
k_{e f}^{M E 1}=k_{s} \frac{2 k_{s}+k_{g}-2\left(k_{s}-k_{g}\right) \cdot \varphi}{2 k_{s}+k_{g}+\left(k_{s}-k_{g}\right) \cdot \varphi}
$$

The second Maxwell-Euken model (ME2) concerns granular medium where solid phase is dispersed in the gas phase:

$$
k_{e f}^{M E 2}=k_{g} \frac{2 k_{g}+k_{s}-2\left(k_{g}-k_{s}\right) \cdot(1-\varphi)}{2 k_{g}+k_{s}+\left(k_{g}-k_{s}\right) \cdot(1-\varphi)}
$$

Compared to the inequality (4), ME models allow for better evaluation of the range of true effective conductivity of the porous medium:

$$
k_{e f}^{S} \leq k_{e f}^{M E 2} \leq k_{e f} \leq k_{e f}^{M E 1} \leq k_{e f}^{P}
$$

Relation (9) is termed Hashin-Shtrikman inequality [15].

EMT (Effective Medium Theory) was developed through analysis of thermal conduction in the medium with random distribution of solid phase and gas phase $[16,17]$. Contrary to ME models, EMT model concerns the medium where both phases are mutually dispersed. The equation of the effective thermal conductivity of the porous medium in this model is:

$$
\begin{aligned}
& k_{e f}^{E M T}=0.25\left((3 \varphi-1) k_{g}+\left(3 v_{s}-1\right) k_{s}+\right. \\
& \left.\sqrt{\left((3 \varphi-1) k_{g}+\left(3 v_{s}-1\right) k_{s}\right)^{2}+8 k_{g} k_{s}}\right)
\end{aligned}
$$

Another effective thermal conductivity models subjected for consideration are models by
Woodside-Messmer (WM) [18], Assad (AS) [19] and Bruggeman (BR) [20]. Effective thermal conductivity in these models is described with the following equations:

$$
\begin{gathered}
k_{e f}^{W M}=k_{s}^{\varphi} \cdot k_{g}^{(1-\varphi)}=k_{g}\left(\frac{k_{s}}{k_{g}}\right)^{\varphi} \\
k_{e f}^{A S}=k_{s}\left(\frac{k_{g}}{k_{s}}\right)^{\varphi} \\
k_{e f}^{B R}=0,25\left[\left(3 v_{s}-1\right) k_{s}+\left(2-3 v_{s}\right) k_{g}\right]+0,25 k_{g} \sqrt{\Delta}
\end{gathered}
$$

where:

$$
\begin{gathered}
\Delta=\left(\left(3 v_{s}-1\right)^{2} \kappa^{2}+\left(2-3 v_{s}\right)^{2}+2\left(2+9 v_{s}-9 v_{s}^{2}\right) \kappa\right)(14) \\
\kappa=k_{s} / k_{g}
\end{gathered}
$$

Besides the analytical models, effective thermal conductivity of porous media is determined also based on the whole series of numerical models. These models are most often based on the methods: finite elements method, finite difference method or Monte Carlo [21-23]. Development of these techniques is connected with increasingly greater computational power of computers. However, the analytical models still are very popular, which results from its simplicity and the low cost of calculations. Analysis of the problem in this article is limited only to the listed analytical models.

\section{Measurements of Effective Thermal Conductivity of Section Bundles}

Evaluation of the usefulness of specific analytical models for determination of the effective thermal conductivity $k_{\mathrm{ef}}$ of section bundles requires knowledge of real values of this parameter. The most reliable information on this topic provides the experimental measurements. Research on measurements of the effective thermal conductivity of steel section bundles are presented in the paper [24]. These measurements were performed by the steady state method. For this purpose was used the guarded hot plate apparatus $[25,26]$. 
The idea of the effective thermal conductivity measurement in relation to the bundles of rectangular sections is shown in Fig. 2. As can be seen the tested samples have the form of flat beds of sections. The measurement procedure involves forcing in the sample a unidirectional steady heat flow, which is expressed quantitatively by heat flux $q$. After obtaining in the system a steady state, the temperatures on the bottom surface (which has temperature $t_{\mathrm{B}}$ ) and the upper surface (which has temperature $t_{\mathrm{U}}$ ) of the sample were measured. The value of $k_{\text {ef }}$ coefficient is calculated from the equation:

$$
k_{e f}=\frac{q \cdot h}{t_{B}-t_{U}}
$$

where $h$ is the dimension of the sample in the direction of heat flow.

Tested samples were made of four different types of sections which have the following dimensions: $20 \times$ $40 \mathrm{~mm}, 40 \times 40 \mathrm{~mm}, 60 \times 60 \mathrm{~mm}$ and $80 \times 80 \mathrm{~mm}$. The wall thickness of the sections $20 \times 40 \mathrm{~mm}$ was 2 $\mathrm{mm}$, for the remaining sections it was $3 \mathrm{~mm}$. For each type of the sections were investigated two samples, marked as $I$ and $I I$. The investigations were performed for the temperature range of $50 \div 600{ }^{\circ} \mathrm{C}$.

The measurement results for the samples made from the sections $20 \times 40 \mathrm{~mm}$ and $40 \times 40 \mathrm{~mm}$ are shown on the Fig. 3a, whereas Fig. $3 b$ shows the results for the samples made from the sections $60 \times 60$ $\mathrm{mm}$ and $80 \times 80 \mathrm{~mm}$. The symbols of the samples in these graphs represent the height of the profiles expressed in mm. Values of the coefficient $k_{\text {ef }}$ obtained for the samples of sections $20 \times 40 \mathrm{~mm}$ do not show significant changes with respect to temperature and range from $3.6 \div 3.9 \mathrm{~W} /(\mathrm{m} \cdot \mathrm{K})$. Mean value of the

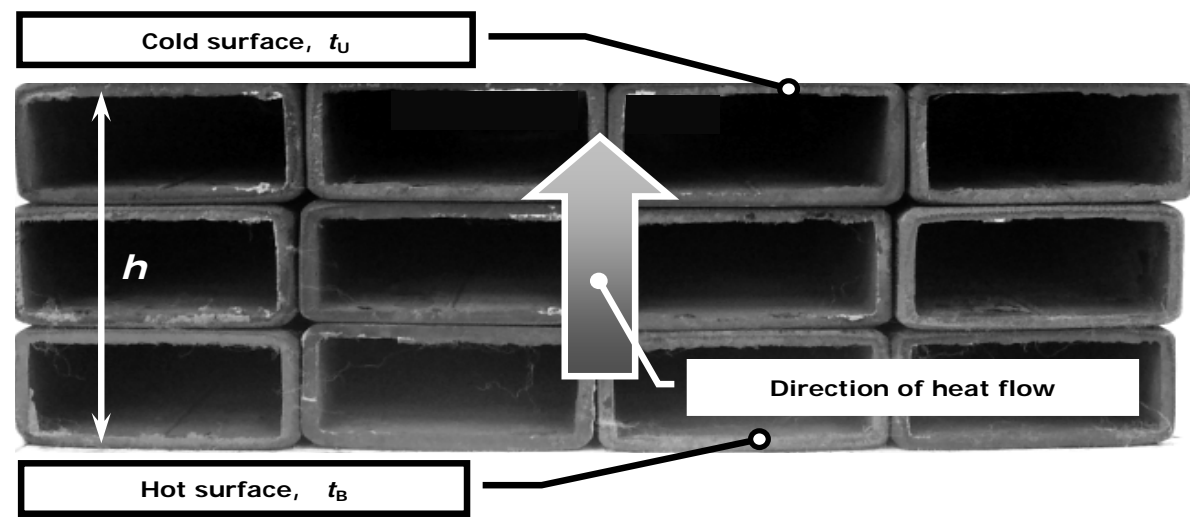

Fig. 2 The idea of the effective thermal conductivity measurement of the flat bed formed from rectangular sections.

(a)

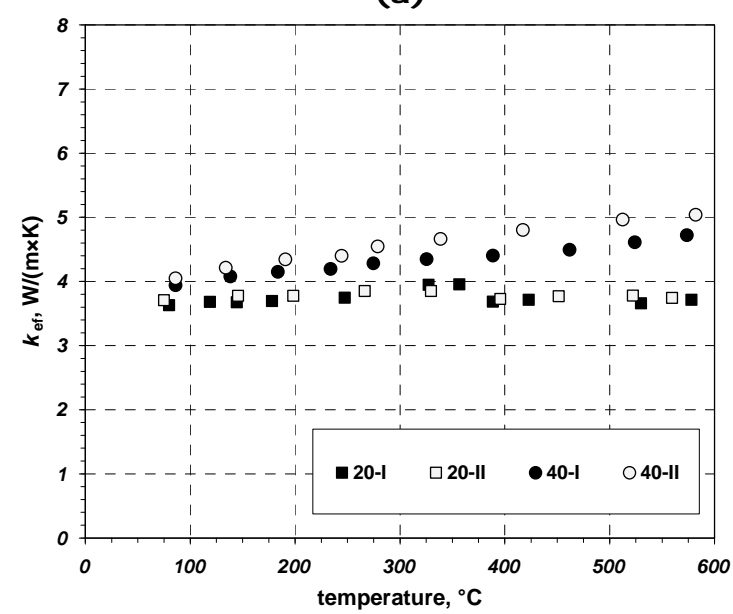

(b)

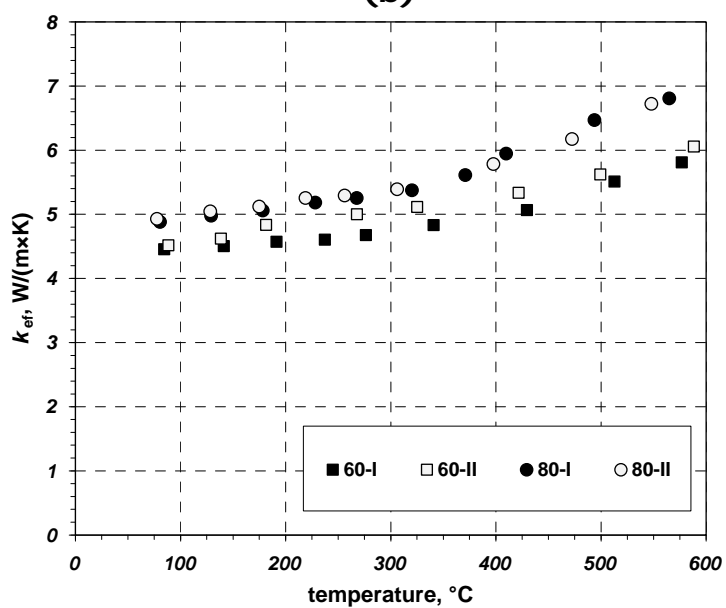

Fig. 3 Results of measurements of the effective thermal conductivity obtained for the samples of the sections (a) $20 \times 40 \mathrm{~mm}$ and $40 \times 40 \mathrm{~mm}$, (b) $60 \times 60 \mathrm{~mm}$ and $80 \times 80 \mathrm{~mm}$. 
$k_{\text {ef }}$ coefficient for these samples in the studied temperature range is $3.75 \mathrm{~W} /(\mathrm{m} \cdot \mathrm{K})$. Effective thermal conductivity for other specimens is increasing with temperature. For the samples of sections $40 \times 40 \mathrm{~mm}$, the obtained values are $3.9 \div 5.1 \mathrm{~W} /(\mathrm{m} \cdot \mathrm{K})$. Furthermore, for the profiles of $60 \times 60 \mathrm{~mm}$ and $80 \times 80 \mathrm{~mm}$, measured values of coefficient $k_{\text {ef }}$ ranged from $4.4 \div 6.1$ $\mathrm{W} /(\mathrm{m} \cdot \mathrm{K})$ and $4.9 \div 6.8 \mathrm{~W} /(\mathrm{m} \cdot \mathrm{K})$. The differences between the results obtained for samples $I$ and II made of sections of the same type are insignificant and are held within the uncertainty of measurement, which in the used stand is of 5\% [27]. In general, it can be concluded that the value of the coefficient $k_{\mathrm{ef}}$ in all examined samples range from $3.2 \div 6.8 \mathrm{~W} /(\mathrm{m} \cdot \mathrm{K})$. These values are by an order of magnitude lower than thermal conductivity of the solid phase (steel) of the investigated bundles.

Furthermore, the presented data show that effective conductivity is greater for samples from bigger sections. This means that the effective thermal conductivity is increasing for the bundles of higher porosity. This is due to the fact, that internal porosity of the section increase when enlarge its external dimensions.

\section{Results}

It was adopted during calculation of the $k_{\mathrm{ef}}$ coefficient that thermal conductivity of the solid phase $k_{\mathrm{s}}$ and gas phase $k_{\mathrm{g}}$ of the bundles with respect to temperature $t$ (expressed in ${ }^{\circ} \mathrm{C}$ ), change according to the following equations:

$$
\begin{gathered}
k_{s}=1.24 \cdot 10^{-8} \cdot t^{3}-3.26 \cdot 10^{-5} \cdot t^{2}- \\
1.19 \cdot 10^{-2} \cdot t+51.35 \\
k_{g}=-2.882 \cdot 10^{-8} \cdot t^{2}+8.051 \cdot 10^{-5} \cdot t+0.024
\end{gathered}
$$

These equations were determined through approximation of the tabular literature data $[6,28]$. Eq. (17) describes changes in thermal conductivity of low-alloy steel with carbon content equal $0.2 \%$. Profiles from this steel were used during experimental studies. Furthermore, Eq. (18) describes changes in thermal conductivity of air. Calculations for each model were performed for the range of temperatures of $25 \div 700{ }^{\circ} \mathrm{C}$.

Another parameter taken into account was porosity $\varphi$, for which three values were adopted: $0.75,0.84$ and 0.88 . Porosity of 0.75 concern to the bundles of section $20 \times 40 \mathrm{~mm}$ with wall thickness of $2 \mathrm{~mm}$ and bundles of section $40 \times 40 \mathrm{~mm}$ with wall thickness of $3 \mathrm{~mm}$. Values of porosity of 0.84 and 0.88 concern respectively to the bundles of section of $60 \times 60 \mathrm{~mm}$ and $80 \times 80 \mathrm{~mm}$ with wall thickness of $3 \mathrm{~mm}$. The adopted values of the parameter $\varphi$ include the external porosity of 0.03 .

Presentation of the calculation data was started from the results of layered models which were presented in Fig. 4. Fig. 4a concerns the parallel model (P), whereas Fig. $4 \mathrm{~b}$ concerns the series model (S). Values of coefficient $k_{\mathrm{ef}}$ obtained for the model $\mathrm{P}$ substantially depend on porosity and decrease as a function of this parameter. Coefficient $k_{\text {ef }}$ obtained in this model also decreases with respect to temperature, which is caused by conduction in the solid phase-according to Eq. (17) the thermal conductivity of $k_{\mathrm{s}}$ decreasing with temperature. The effective conductivity for this model ranges from $4.4 \div 12.8$ $\mathrm{W} /(\mathrm{m} \cdot \mathrm{K})$. On the one hand, this order of magnitude is comparable with the measurement data. However, the accurate comparison of both results shows that the parallel model does not reflect both tendencies for changes in the coefficient $k_{\text {ef }}$ as well as its values, which are doubled in this case.

The effective thermal conductivity obtained for the series model completely does not reflect the actual data. Values of the coefficient $k_{\mathrm{ef}}$ obtained for this model range from $0.03 \div 0.08 \mathrm{~W} /(\mathrm{m} \cdot \mathrm{K})$, i.e. are by two orders of magnitude lower than the values obtained during measurements.

Fig. 5 presents the results obtained for models that represent mean results obtained for parallel and series model. Fig. 5a shows the results obtained for Horai 
(a)

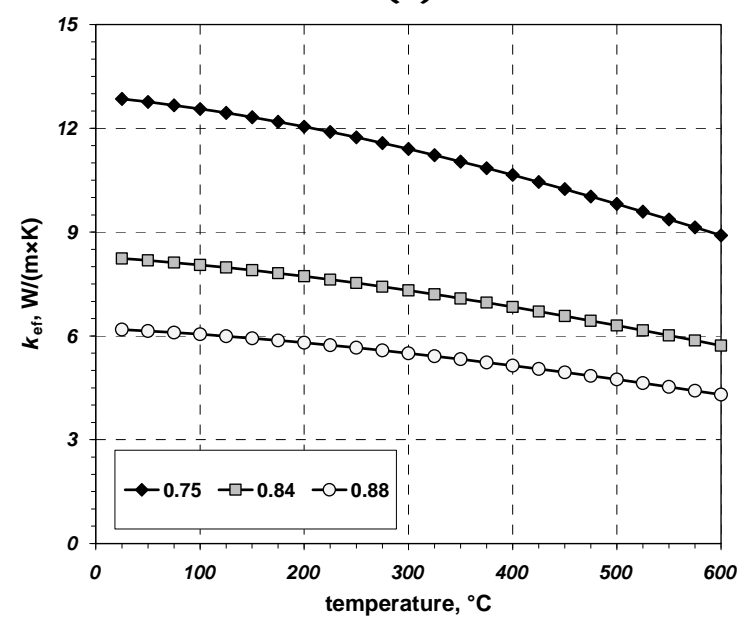

(b)

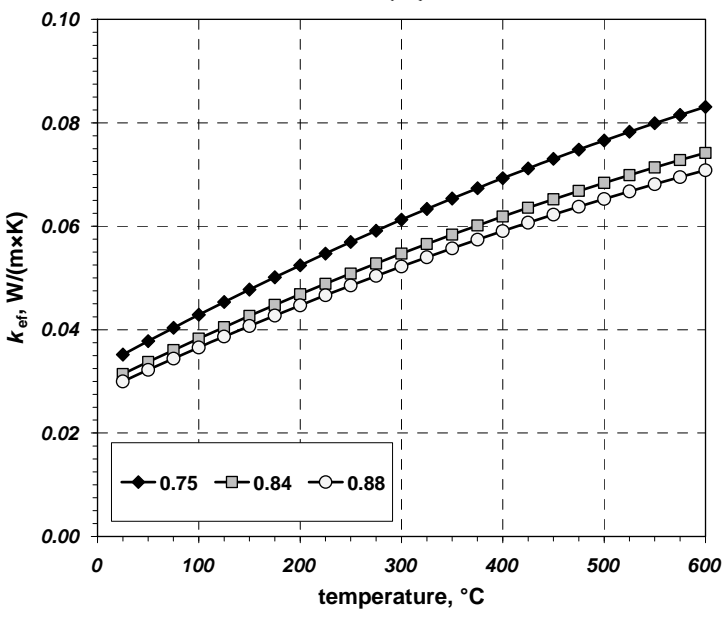

Fig. 4 Effective thermal conductivity of section bundles calculated for: (a) parallel model (P), (b) series model (S).

(a)

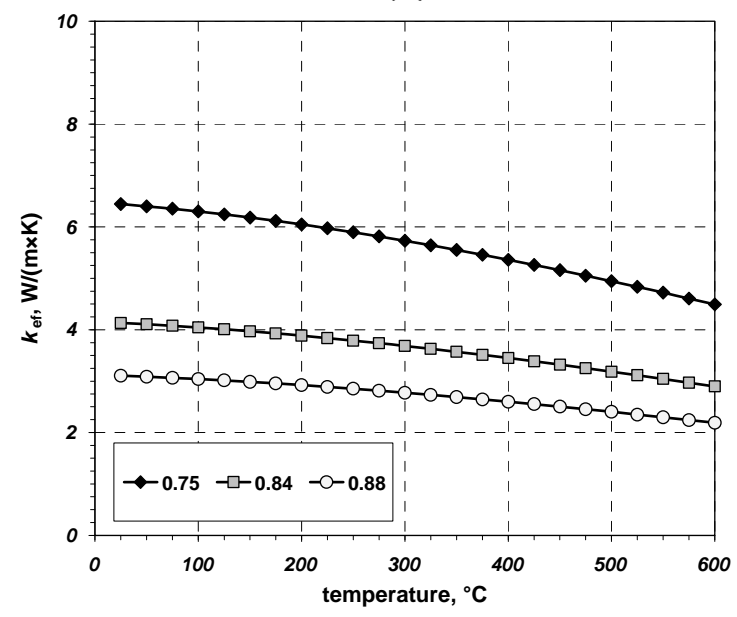

(b)

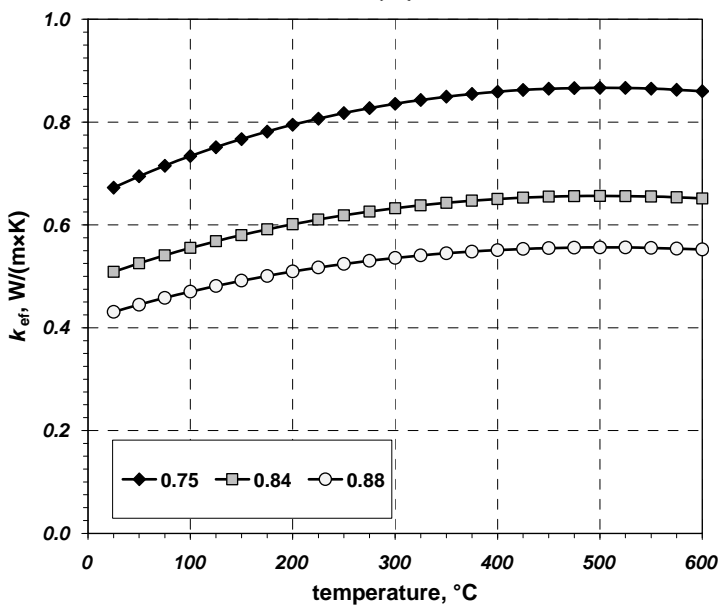

Fig. 5 Effective thermal conductivity of section bundles calculated for: (a) Horai model (H), (b) Beck model (B).

model $(\mathrm{H})$, which represents arithmetic mean of models $\mathrm{P}$ and $\mathrm{S}$. Since insignificant values of the coefficient $k_{\text {ef }}$ were obtained for model S, the results obtained for the model $\mathrm{H}$ are much similar to the model $\mathrm{P}$, with the values ranging from $2.2 \div 6.4$ $\mathrm{W} /(\mathrm{m} \cdot \mathrm{K})$. It can be concluded that this range is similar to the experimental results. However, for model $\mathrm{H}$, there is a opposite tendency of changes in the coefficient $k_{\mathrm{ef}}$ as a function of temperature.

Fig. $5 \mathrm{~b}$ presents the results obtained for Beck's model (B), which is the geometric mean of models $\mathrm{P}$ and S. Coefficient $k_{\text {ef }}$ in this case is rising with the temperature and depends substantially on porosity. The values obtained in this case range from $0.43 \div$
$0.86 \mathrm{~W} /(\mathrm{m} \cdot \mathrm{K})$. This is much below the real values of $k_{\mathrm{ef}}$ coefficient for the discussed medium.

The results presented in Fig. 6 concern Krischer model. The values of coefficient $k_{\text {ef }}$ presented in Fig. $6 \mathrm{a}$ were obtained for the coefficient $f=0.25$. The results presented in Fig. $6 \mathrm{~b}$ were obtained for $f=0.5$. As can be seen, the increase in the parameter $f$ leads to a reduction in the value of coefficient $k_{\text {ef. }}$. Results of calculations compared to the measurement data are reduced by an order of magnitude, for $f=0.25 k_{\mathrm{ef}}$ they range from $0.12 \div 0.32 \mathrm{~W} /(\mathrm{m} \cdot \mathrm{K})$, and, for $f=0.5$, this means ca. $0.1 \mathrm{~W} /(\mathrm{m} \cdot \mathrm{K})$. Theoretically, there is the opportunity to match the optimum value of coefficient $f$, for which the results of calculations would be fully 
(a)

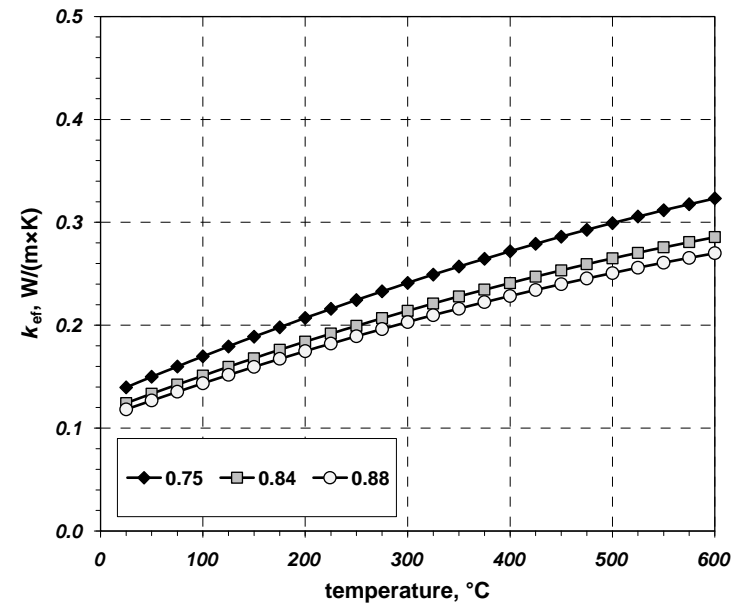

(b)

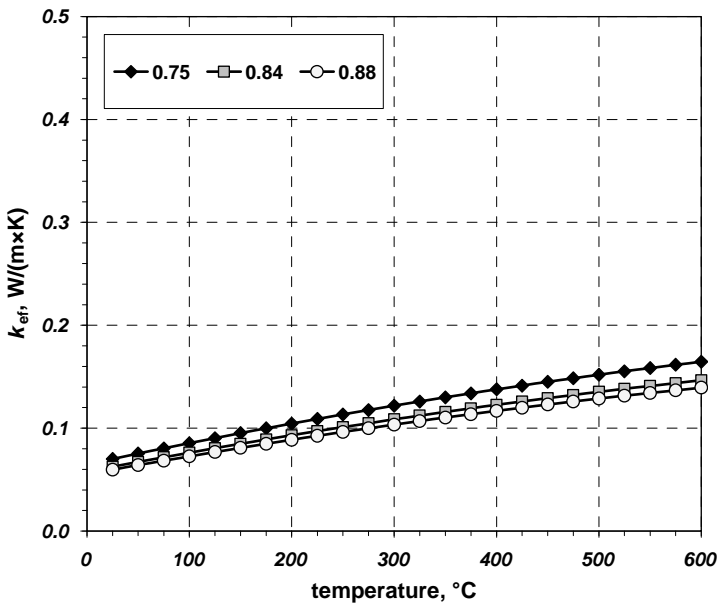

Fig. 6 Effective thermal conductivity of section bunches obtained for the Krischer model: (a) results for $f=0.25$, (b) results for $f=0.5$.

(a)

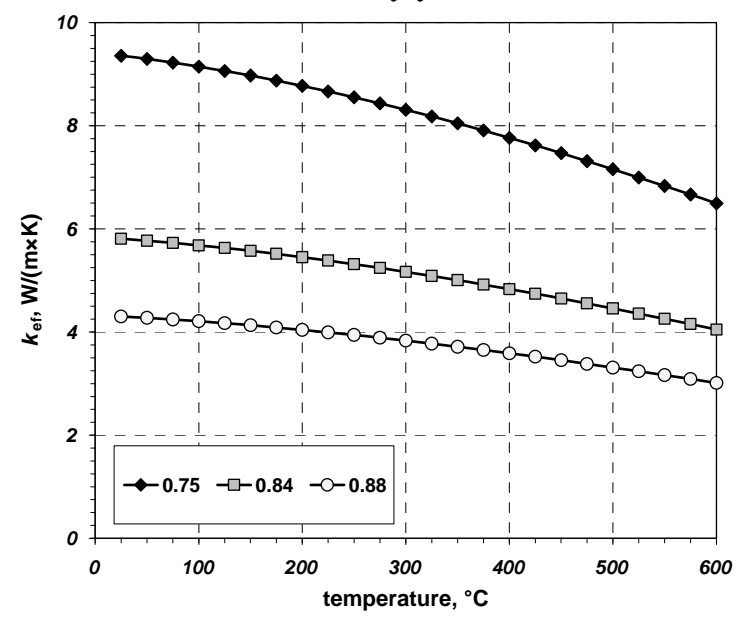

(b)

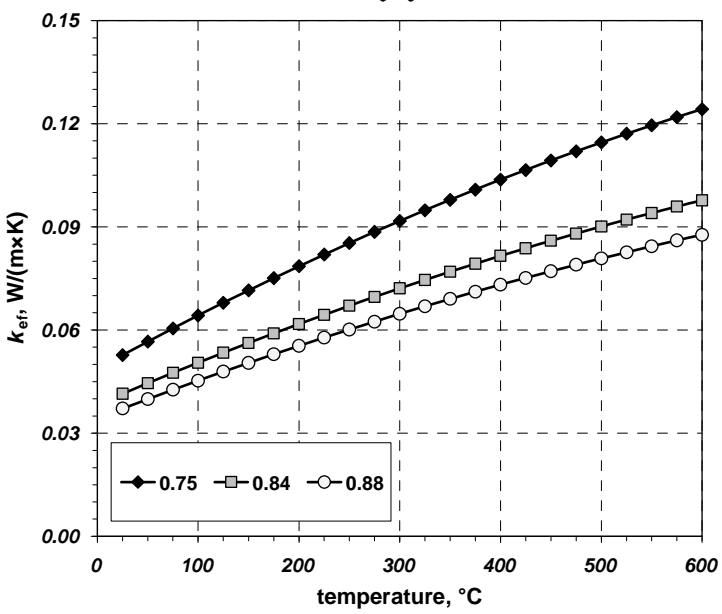

Fig. 7 Effective thermal conductivity of section bundles calculated for Maxwell-Euken models: (a) results for cellular medium - model ME1, (b) results for granular medium - model ME2.

consistent with the experimental data, but obtaining of this match requires multiple calculations. However, if measurement data are unavailable, this match is not possible at all.

Fig. 7 presents the results obtained for Maxwell-Euken (ME) models. The results obtained in Fig. 7a concern the cellular medium (model ME1) with continuous solid phase. The changes of the coefficient $k_{\text {ef }}$ for this model are similar to the results obtained for the parallel model. The difference between these models consists in that the values for the model ME1 are by ca. $40 \%$ lower than the values for the model $\mathrm{P}$. The range of values for the coefficient $k_{\mathrm{ef}}$ of the model ME1 is $3.0 \div 9.4 \mathrm{~W} /(\mathrm{m} \cdot \mathrm{K})$.

The results obtained for the model ME2 are presented in Fig. 7b. As can be seen, the values of coefficient $k_{\text {ef }}$ in this case range from $0.04 \div 0.12$ $\mathrm{W} /(\mathrm{m} \cdot \mathrm{K})$. This result is by two orders of magnitude lower than the actual values.

Fig. 8a illustrates the results obtained for the EMT model. In this model, the increase in porosity (in the range of values adopted here for this parameter) leads to the increase in effective thermal conductivity of the medium. This is the first model for which this tendency was observed. In the case of previously analyzed models, the increase in porosity contributes 
(a)

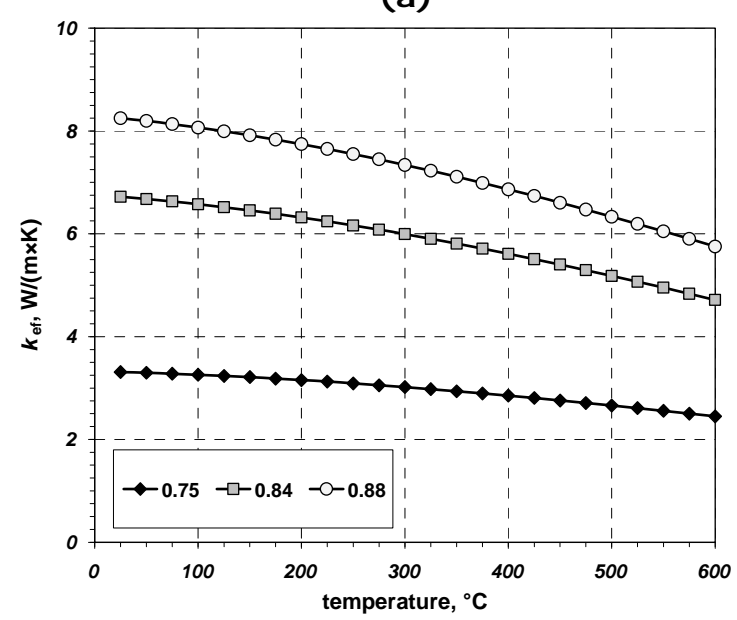

(b)

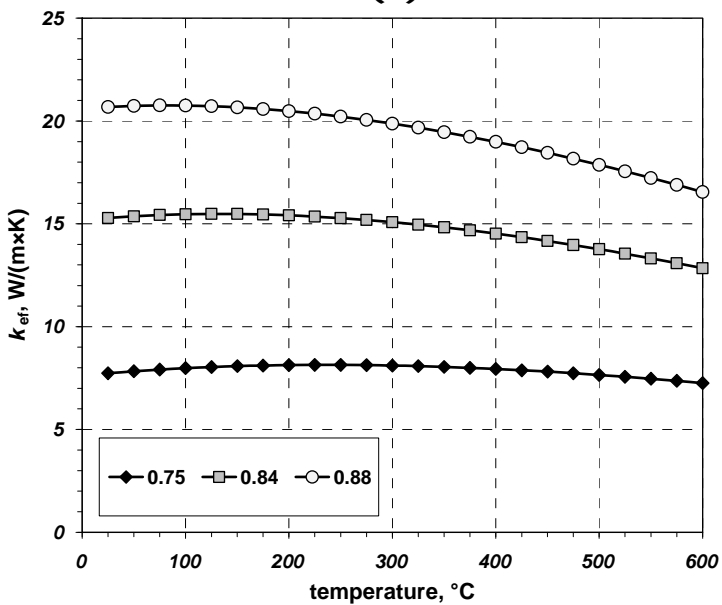

Fig. 8 Effective thermal conductivity of section bundles calculated for: (a) EMT model, (b) Woodside-Messmer model (WM).

(a)

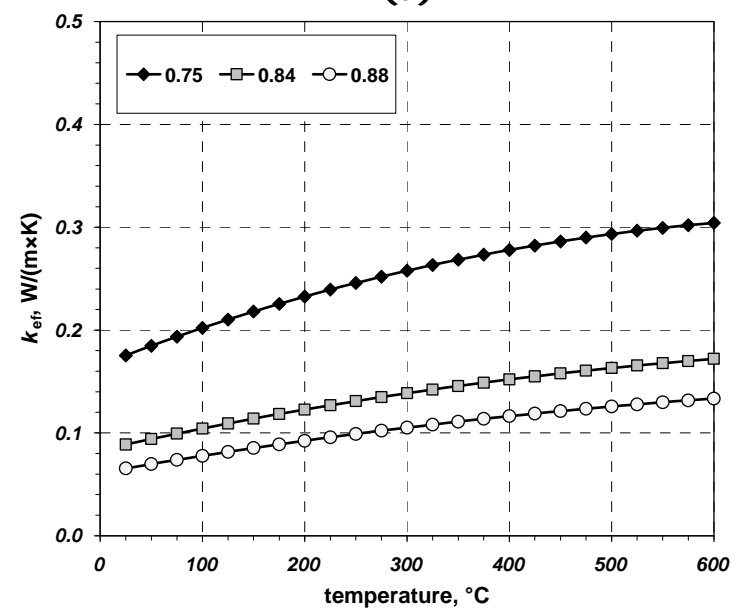

(b)

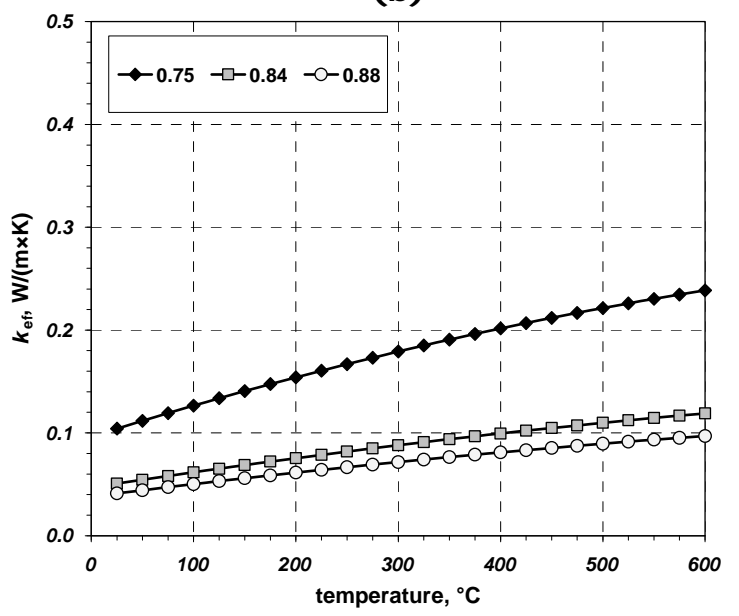

Fig. 9 Effective thermal conductivity of section bundles calculated for: (a) Assad model (A), (b) Bruggeman model (BR).

to the reduction in the value of the coefficient $k_{\text {ef. }}$ As was demonstrated by the measurements, the increase in porosity in real section bundles is also correlated with the increase in coefficient $k_{\text {ef. }}$. Therefore, it can be adopted that the EMT model with respect to porosity reflects correct changes in effective thermal conductivity of the sections. Furthermore, the values of $k_{\mathrm{ef}}$ obtained in this case, ranging from $2.5 \div 8.2$ $\mathrm{W} /(\mathrm{m} \cdot \mathrm{K})$ are comparable with the measurement data. However, effective conductivity of bundles calculated for EMT model decreases with temperature, which is the opposite of the tendencies observed in the real medium. This causes that this model also does not reflect the effective thermal conductivity of the analyzed charge. Theoretically, if the tendency of changes in the coefficient $k_{\mathrm{ef}}$ with respect to temperature would be reversed, this model (for the studied range of porosity) would nearly perfectly reflect thermal properties of the considered medium. Since this is not the case, the observed similarity of results has entirely random character.

Fig. $8 \mathrm{~b}$ illustrates the results obtained for Woodside-Messmer model (WM). Comparison of the data from Figs. $8 \mathrm{a}$ and $8 \mathrm{~b}$ shows that the changes in coefficient $k_{\mathrm{ef}}$ obtained for models EMT and WM are similar to some degree. However, the values obtained 
for WM model are by ca. two and half times greater than the values of the EMT model. This means that, besides the drawbacks mentioned during discussion on the results obtained for the EMT model, the model WM shows exaggerated values of the analyzed parameter.

Fig. 9 presents the results obtained for two last models: Asaad (A) and Bruggeman (BR). As can be noted, both models give similar results. The values of the coefficient $k_{\mathrm{ef}}$ for these models are by an order of magnitude lower than the real values. Values of the coefficient $k_{\text {ef }}$ calculated according to the Assad model (Fig. 9a) range from $0.06 \div 0.3 \mathrm{~W} /(\mathrm{m} \cdot \mathrm{K})$, whereas for the Bruggeman's model, this is $0.04 \div 0.24 \mathrm{~W} /(\mathrm{m} \cdot \mathrm{K})$. Therefore, both models cannot be used for modelling of thermal properties of bundles of rectangular steel sections.

\section{Conclusion}

With regard for the values of $k_{\text {ef }}$ coefficient for bundles of rectangular steel profiles obtained in the experimental investigations, one should find that none of the eleven models of effective thermal conductivity is suitable for evaluation of thermal properties of this charge. This happens because the models used in the study take into consideration only thermal conduction that occurs in particular phases of the medium. During heating of the bundles, apart from heat conduction in the steel and gas phase, contact conduction in the locations of contact with adjacent sections, thermal radiation between the surfaces of sections and gas convection inside the sections can be observed. The models analyzed in the study do not take these phenomena into consideration. Consequently in the case of the medium with such complexity of the heat flow, the results obtained are worthless. Although the value of coefficient $k_{\text {ef }}$ obtained by means of models: parallel, Horai, Maxwell-Euken for the cellular medium (ME1), EMT and Woodside-Messmer show similar order of magnitude compared to the experimental data, but none of the models reflects the correct changes in the value of coefficient $k_{\mathrm{ef}}$ with respect to temperature. With respect to model calculations, coefficient $k_{\text {ef }}$ decreases with the increase in temperature, whereas it actually increases as a function of this parameter. Therefore, the similarity of the value of coefficient $k_{\text {ef }}$ obtained using these models should be regarded as coincidental.

The obtained results demonstrated that analytical calculation of the effective thermal conductivity of steel rectangular section bundles necessitates building an entirely new model. This model should take into consideration all the mechanisms of heat transfer that occur in this medium. Development of this model represents another stage of the research work carried out by the author in this field.

\section{References}

[1] Mehta, R., and Sahay, S. S. 2009. "Heat Transfer Mechanism and Furnace Productivity during Coil Annealing: Aluminum vs. Steel." Journal of Materials Engineering and Performance 1: 8-15.

[2] Sahay, S., Mehta, R., and Krishnan, K. 2007. "Genetic-Algorithm-Based Optimization of an Industrial Age-Hardening Operation for Packed Bundles of Aluminum Rods." Materials and Manufacturing Processes 22: 615-22.

[3] Jaluria, Y. 1988. "Numerical Simulation of the Transport Process in a Heat Treatment Furnace." International Journal for Numerical Methods in Engineering 25: 387-99.

[4] Rao, T. R., Barth, G. J., and Miller J. R. 1983. "Computer Model Prediction of Heating, Soaking and Cooking Times in Batch Coil Annealing." Iron and Steel Engineering 60: 22-33.

[5] Jacobsen, R. T., and Wright N. T. 2003. "Thermphysical Properties of Fluids and Materials." In Heat Transfer Handbook, edited by Bejan, A., and Kraus, A. D. New York: John Willey and Sons.

[6] Raźnjević, K. 1966. Thermal Table with Diagrams. Warsaw: WNT. (in Polish)

[7] Kaviany, M. 1995. Principles of Heat Transfer in Porous Media, 2nd ed. New York: Springer-Verlag.

[8] Kwon, J-S., Jang, Ch. H., Jung, H., and Song, T-H. 2009. "Effective Thermal Conductivity of Various Filling Materials for Vacuum Insulation Panels." International Journal of Heat and Mass Transfer 52: 5525-32.

[9] Singh, R. 2008. "Thermal Conduction through Porous Systems." In Cellular and Porous Materials: Thermal 
Properties Simulation and Prediction. WILEY-VCH Verlag GmbHandCo, 199-238.

[10] Van Antwerpen, W., du Toit, C. G., and Rousseau, P. G. 2010. "A Review of Correlations to Model the Packing Structure and Effective Thermal Conductivity in Packed Beds of Mono-Sized Spherical Particles." Nuclear Engineering and Design 240: 1803-18.

[11] Carson, J. K. 2005. "Thermal Conductivity Bounds for Isotropic Porous Materials." International Journal Heat and Mass Transfer 48: 2150-8.

[12] Horai, K. 1991. "Thermal Conductivity of Hawaiian Basalt: A New Interpretation of Robertson and Peck's Data.” Journal of Geophysics Res 96: 4125-32.

[13] Beck, J. M., and Beck, A. E. 1965. "Computing Thermal Conductivities of Rocks from Chips and Conventional Specimen." Journal of Geophysics Res 70: 5227-39.

[14] Krischer, O. 1963. Die wissenschlaftlichen Grundlagen der Trocknungstechnik. Berlin: Springer-Verlag.

[15] Hashin, Z., and Shtrikman, S. 1962. "A Variational Approach to the Theory of the Effective Magnetic Permeability of Multiphase Materials." Journal of Applied Physics 33: 3125-31.

[16] Landauer, R. 1952. "The Electrical Resistance of Binary Metallic Mixture." Journal of Applied Physics 23: 779-84.

[17] Pietrak, K., and Wiśnewski, T. S. 2015. "A Review of Models for Effective Thermal Conductivity of Composite Material." Journal of Power Technologies 1: 14-24.

[18] Woodside, W., Messmer, J. 1961. "Thermal Conductivity of Porous Media, I: Unconsolidated Sands and II: Consolidated Sands.” Journal of Applied Physics 32: 1688-768.

[19] Assad, A. 1955. Study of Thermal Conductivity of Fluid Bearing Porous Rocks. University of California, California,

[20] Abareshi, M., and Youssefi, A. 2010. "Fabrication, Characterization and Measurement of Thermal Conductivity of $\mathrm{Fe}_{3} \mathrm{O}_{4}$ Nanofluids." Journal of
Magnetism and Magnetic Materials 322: 3895-901.

[21] Belova, I. V., and Murch, G. E. 2008. "Thermal Properties of Composite Materials and Porous Media: Lattice-Based Monte Carlo Approaches.” In Cellular and Porous Materials: Thermal Properties Simulation and Prediction. WILEY-VCH Verlag GmbHandCo, 73-96.

[22] Floury, J., Carson, J., and Pham, Q. T. 2008. "Modelling Thermal Conductivity in Heterogeneous Media with the Finite Element Method.” Food Bioprocess Technology 1: 161-170.

[23] Oschner, A., and Fiedler, T. 2008. "Effective Thermal Properties of Hollow-Sphere Structures: A Finite Element Approach." In Cellular and Porous Materials: Thermal Properties Simulation and Prediction. WILEY-VCH Verlag GmbHandCo, 31-72.

[24] Wyczolkowski, R., and Benduch, A. 2014. "The Experimental Study of the Effective Thermal Conductivity of Bundles of Rectangular Steel Sections." Civil and Environmental Engineering Reports 14 (3): 119-32.

[25] ASTM C1044-12. 2012. Standard Practice for Using a Guarded-Hot-Plate Apparatus or Thin-Heater Apparatus in the Single-Sided Mode. ASTM International, West Conshohocken, PA.

[26] ASTM C177-13. 2013. Standard Test Method for Steady-State Heat Flux Measurements and Thermal Transmission Properties by Means of the Guarded-Hot-Plate Apparatus. ASTM International, West Conshohocken, PA.

[27] Benduch, A., and Wyczolkowki, R. 2015. "Analysis of the Measurement of the Determining the Effective Thermal Conductivity with the Use of Guarded Hot Plate Apparatus." XVI International Scientific Conference "New Technologies and Achievements in Metallurgy, Material Engineering and Production Engineering, Monographs No. 48, 381-8, Czestochowa. (in polish)

[28] Senkara, T. 1981. Thermal Calculations of Furnaces in Metallurgy. Katowice: Silesia Publisher. (in polish) 